Artículo de reflexión

\title{
La concurrencia de la libertad transcendental y la causalidad natural en la filosofía kantiana ${ }^{1}$
}

\author{
The concurrence of transcendental freedom and \\ natural causality in Kantian philosophy
}

Fernando García Martín ${ }^{2}$

Universidad Nacional de Educación a Distancia, España

Recepción: 10 de diciembre del 2020

Evaluación: 20 de marzo del 2021

Aceptación: 26 de marzo del 2021

\footnotetext{
1 Artículo redactado durante el proceso de elaboración de la tesis doctoral El problema de la libertad y su solución en el marco de la filosofía kantiana, en la Facultad de Filosofía de la Universidad Nacional de Educación a Distancia (UNED).

2 Licenciado en Ciencias Físicas, Licenciado en Filosofía y Doctor en Filosofía de la Universidad Nacional de Educación a Distancia (UNED).

Correo electrónico: fgarcia1019@alumno.uned.es
} 


\title{
Resumen:
}

La Tercera Antinomia de la $\mathrm{Kr} V$ constituye un buen ejemplo de la tensión que en la cultura moderna se establece entre la libertad y la causalidad natural. Kant procede a solventar el conflicto entre ambas mediante la distinción entre fenómeno y cosa en sí. Pero siempre cabrá la duda de si esa distinción no consistirá en un intento ad hoc por salvar la realidad de la libertad de los ataques de índole determinista a los que es sometida y que dan lugar a que muchos suscriban un concepto compatibilista de ella. En el presente artículo se esboza una interpretación de la categoría de causalidad natural kantiana, en términos de principio regulativo de la investigación científica, que permita la defensa de un espacio ontológico para la realidad de la libertad, sin tener para ello que adoptar una posición incompatibilista, y para que sea al mismo tiempo coherente con los resultados que nos ofrecen las modernas investigaciones cuánticas.

Palabras clave: Kant, causalidad, libertad, determinismo, física cuántica.

\begin{abstract}
:
The Third Antinomy of the $K r V$ is a good example of the tension that exists in modern culture between freedom and natural causality. Kant proceeds to resolve the conflict between the two by distinguishing between phenomenon and thing-in-itself. But there will always be a doubt as to whether this distinction will not consist of an ad hoc attempt to save the reality of freedom from the deterministic attacks to which it is subjected and which lead many to subscribe to a compatibilist concept of it. This article outlines an interpretation of the Kantian category of natural causality in terms of the regulative principle of scientific research that allows the defense of an ontological space for the reality of freedom without having to adopt an incompatible position and be at the same time consistent with the results that modern quantum research offers us.
\end{abstract}

Keywords: Kant, causality, freedom, determinism, quantum physics. 


\section{Introducción}

Hace aproximadamente 2.500 años Heráclito sostuvo que "la guerra (pólemos) es el padre de todas las cosas" (DK 22 B53), dando pie a una de las modalidades interpretativas acerca de la realidad que más ha sido ensayada en la historia del pensamiento. De acuerdo con ella, lo definitorio de lo real, sea cual sea el enfoque en su consideración (natural, antropológico, social, económico, político, etc.), vendría a ser el continuo enfrentamiento entre diversos elementos en su aspiración a protagonizar toda la realidad, del que podría no obstante resultar su mutua conciliación.

La diversidad de tendencias a través de las cuales la existencia humana se desarrolla, constituye una nota distintiva de nuestra cultura y civilización. Siguiendo el modo heraclíteo de pensar, este carácter polifacético de la realidad puede achacarse a la presencia en ella de diversos pares de conceptos, donde cada uno de sus elementos componentes se muestra como igualmente fundamentado y con idénticas pretensiones de alcanzar la supremacía respecto del otro elemento integrante de la disyunción. Un ejemplo del carácter conflictivo que se da entre algunas ideas configuradoras de nuestro modo de pensar, lo constituye la recíproca relación de oposición que habitualmente se establece entre la idea de libertad y el concepto de causalidad natural, cuando se procede sosteniendo que la afirmación de la realidad de la primera excluye completamente la del segundo o bien lo contrario, es decir, que allá donde se dé la causalidad natural no pueda encontrarse presente la libertad.

La posición que concede a la causalidad natural una presencia ilimitada en la configuración de la realidad, tiene en el "determinismo" una concreción de índole filosófica y científica. Entre las diversas formulaciones que del determinismo se han proporcionado en los últimos siglos, merece ser destacada la que llevó a cabo Laplace en su Ensayo filosófico sobre las probabilidades, al sostener que "(...) hemos de considerar el estado actual del universo como el efecto de su estado anterior y como la causa del que ha de seguirle" (Laplace, 1985, pp. 25-26).

Los espectaculares avances conseguidos en la aplicación al mundo natural del concepto de causalidad natural involucrado en esta forma de determinismo y la defensa de su posibilidad de aplicación a ámbitos tenidos por específicamente humanos, como es el psicológico, han dado lugar a que se plantee la cuestión de si la caracterización que el hombre hace de sí mismo como ser libre no será nada más que una ilusión que él se ha ido forjando 
a lo largo de su historia, dando cuenta más de una tendencia narcisista suya que de una adecuada interpretación de la realidad. En el caso de que así efectivamente fuese, parece entonces que lo más que podría defenderse respecto de la realidad de la libertad humana sería un concepto compatibilista suyo. Conforme con él, puesto que el fundamento último determinante de las acciones humanas siempre sería de índole natural, la atribución de la condición libre a un ser humano concreto no vendría a ser nada más que una especificación aclaratoria de que el motivo de su acción se encuentra en él mismo y no en algo externo a él. En tal caso, ocurriría que los supuestos comportamientos "libres" de los seres humanos no pondrían en entredicho el alcance ilimitado que se concede a la causalidad natural en este concepto de libertad, por lo que se encontraría subordinada en su carácter de realidad a la de la causalidad natural.

La tradición filosófica dispone de varios ejemplos de pensadores que, aun sin adoptar una formulación equiparable a la del determinismo laplaciano en la consideración de la conducta humana, sostuvieron que su fundamento de determinación es de índole exclusivamente natural. Tal es el caso de la postura en relación con la conciencia de la libertad sostenida por Spinoza, para quien:

(...) los hombres se equivocan al creerse libres, opinión que obedece al solo hecho de que son conscientes de sus acciones e ignorantes de las causas que las determinan. Y, por tanto, su idea de "libertad" se reduce al desconocimiento de las causas de sus acciones, pues todo eso que dicen de que las acciones humanas dependen de la voluntad son palabras, sin idea alguna que les corresponda (Spinoza, 2007, p. 157).

El determinismo laplaciano constituye una de las posturas que pueden adoptarse en la discusión en torno al presunto enfrentamiento excluyente entre la causalidad natural y la libertad. Puede plantearse cuál habría de ser la postura que representase el otro extremo posible de este al menos aparente dilema. Esta postura, que calificaremos de "libertarianista", sería aquella que defendiera que la práctica de la libertad supone de manera necesaria la suspensión, al menos momentánea, de la vigencia de la causalidad natural en el mundo. Tal sería el caso de los "milagros" de los que nos habla la tradición religiosa. Ahora bien, para poder llevar a cabo una actuación "milagrosa" en el mundo, que supusiese la suspensión de la validez de sus leyes naturales, quien la llevase a efecto habría de contar con la omnipotencia como uno de sus atributos. Esta situación que no puede ser la situación humana, 
plantea la inviabilidad de esta concepción de la libertad. No obstante, el posicionamiento "libertarianista" también habrá de ser tenido en cuenta en la discusión sistemática en torno a la realidad de la libertad, la de la causalidad $\mathrm{y}$ las relaciones mutuas mantenidas entre una y otra.

Aunque resulta evidente que cualquier discusión relacionada con esta temática no puede dejar de tener en cuenta que su ámbito último de consideración ha de ser necesariamente el de la existencia humana, en el presente artículo no entraremos a valorar la dimensión práctica asociada a la idea de libertad, sino que nos limitaremos al tratamiento de las cuestiones relativas a la realidad de la libertad desde una perspectiva meramente especulativa. Acorde con esta intención, serán tenidas en cuenta, en lo referente al alcance y el modo de validez de la causalidad natural, tanto algunas de las implicaciones que se derivan de los desarrollos científicos clásicos como las que se pueden obtener suscribiendo el nuevo paradigma científico abierto por la moderna física cuántica.

El tratamiento de la temática relativa a las relaciones mantenidas entre la causalidad natural y la libertad, que en el presente artículo nos ocupa, tiene en la Dialéctica de la Crítica de la razón pura kantiana uno de sus textos fundamentales de referencia, por lo que ella será tomada como hilo conductor de las sucesivas reflexiones que plantearemos.

\section{El conflicto entre la libertad y la causalidad natural en la filosofía kantiana: la Tercera Antinomia de la $\mathrm{Kr} V$}

En su conocida carta a Christian Garve del 21 de septiembre de 1798, Kant manifestó que no fue la investigación en torno a la existencia de Dios, o a la inmortalidad del alma, el punto a partir del cual se originó su filosofía crítica, sino que ésta surgió más bien como un intento por resolver el "escándalo de la aparente contradicción de la razón consigo misma" (das Skandal des scheinbaren Widerspruchs der Vernunft mit ihr selbst) al que daba lugar la existencia de una Antinomia de la razón pura ( $A A$, XII 257-258). El carácter inevitable por él atribuido a la disputa antinómica hace de Kant un digno representante de la índole conflictiva e incluso contradictoria propia de la Modernidad y de la condición humana que a partir de ella se configura. En particular, resulta especialmente relevante al respecto el conflicto entre la causalidad natural y la libertad presente en la Tercera Antinomia de la $\mathrm{KrV}$, tomando la forma de enfrentamiento excluyente entre lo sostenido en la postura de la tesis (la posibilidad de la libertad) y lo defendido en la antítesis (la omnipresencia de la causalidad natural). 
La tesis de esta Antinomia establece que "la causalidad según leyes de la naturaleza no es la única de la que pueden derivar los fenómenos todos del mundo. Para explicar éstos nos hace falta otra causalidad por libertad" $(\mathrm{KrV}$, A444/B472).

Esta posición surge por un requerimiento de índole metodológico propio de la investigación científica, consistente en que, para que el estado del mundo sea plenamente conocido, se precisa que todos sus sucesos (Ereignisse, Begebenheiten) hayan sido explicados de manera exhaustiva. Pero la exhaustividad en la explicación de un suceso únicamente puede lograrse recorriendo en su totalidad de manera regresiva la serie de condicionados y condiciones de la que ha resultado, ya que el suceso antecedente de cualquier suceso sólo puede ser conocido considerándolo como condicionado a su vez por otro anterior. Por ello, ante su incapacidad para recorrer completamente la serie ascendente condicionado-condición, la razón se ve obligada a asumir un punto de descanso (Ruhepunkt), es decir, a suponer que hay una condición que es no-condicionada. Esta condición no-condicionada se corresponde con la idea de causalidad por libertad contenida en el concepto de libertad transcendental, caracterizado por Kant como “(...) una absoluta espontaneidad causal que inicie por sí misma una serie de fenómenos que se desarrollen según leyes de la naturaleza" ( $K r V$, A446/B474).

En relación con las interpretaciones posibles del modelo de causalidad natural, que podrían efectuarse partiendo del texto correspondiente a la tesis de esta antinomia, debe hacerse constar que el objetivo contra el que ella se dirige es el concepto de causalidad de alcance ilimitado, implicado por el determinismo. Estimamos que el motivo de la refutación a la que éste es sometido se encuentra en que, para que su pretensión omnicomprensiva pudiese ser sostenida, se precisaría poder conocer con absoluta precisión el estado del mundo en un momento determinado, lo que implicaría la determinación absoluta de todas las relaciones recíprocas de causalidad entre sus elementos componentes, siendo ello posible sólo si pudiese recorrerse en sentido ascendente la serie completa de condiciones de todos los sucesos o eventos en el mundo, lo que no resulta factible para un ser de capacidades limitadas como es el hombre, por lo que este concepto de causalidad "determinista" ha de ser rechazado. La antítesis sostiene que "no hay libertad. Todo cuanto sucede en el mundo se desarrolla exclusivamente según leyes de la naturaleza" (A445/B473). 
Esta postura surge también por un requerimiento de índole metodológico que se plantea en cualquier investigación que aspire a ser científica. En este caso, él consiste en la exigencia de que en la explicación no debe salirse del ámbito en el que se encuentra lo que se pretende explicar. La 'Estética y la Analítica transcendental' de la $K r V$ han determinado el ámbito en el que ha de llevarse a cabo la explicación de los fenómenos, que es el de su espacio-temporalidad y el de su necesario sometimiento a las categorías en general, y a la categoría de causalidad natural en particular. Ir más allá de este ámbito supondría abandonar la "lógica de la verdad" (Logik der Wahrheit) y entrar en el terreno de la "lógica de la apariencia" (Logik des Scheins) propio de las explicaciones metafísicas, dando lugar a la infracción de lo metodológicamente legítimo que nuestro autor denuncia en la Observación hecha a la postura de la antítesis, por cuanto supondría el cuestionamiento de la sistematicidad misma del conocimiento (A449-452/B477-480).

Si ahora se atiende a cuál es el objetivo perseguido al enunciar esta antítesis, debe indicarse que él podría consistir en la refutación del concepto de libertad resultante de la postura calificada en el presente artículo como "libertarianista", pues la suspensión temporal de la vigencia de la categoría de causalidad natural (que según esta postura va unida ineludiblemente a cualquier práctica realmente libre) daría lugar a la ruptura en la serie de la experiencia (Erfahrung).

Las Antinomias dejan a la razón "en un estado de permanente vacilación" (in einem unaufhörlich schwankenden Zustande), pues ésta no sabe qué postura es la que tendrá que adoptar, si habrá de ser la de las tesis o bien la de las antítesis (A475/B503). En las dos primeras Antinomias (antinomias matemáticas) al menos el conflicto quedaba limitado al orden de lo teórico. Pero en las otras dos (antinomias dinámicas) no queda reducido a este orden, sino que toma más bien la forma de pugna entre el orden de lo teórico y el de lo práctico. Particularmente en la Tercera Antinomia la afirmación o la negación de otro tipo de causalidad, como lo es la causalidad por libertad, no es sólo relevante dentro del ámbito orientado al conocimiento (Erkenntniß), sino también en el dirigido hacia la acción (praxis). Lo primero ocurre porque la aceptación o no de la libertad como un tipo de causalidad que complemente o sea alternativo a la causalidad natural, incide sobre la imagen que se tenga del mundo desde su consideración física. Pero es relevante ante todo en lo referente a lo segundo, pues aquí no puede resultar indiferente de cara a la conducta humana sostener que ésta es libre, o al menos que puede serlo, que decir que no lo es en absoluto. 
El problema de la realidad o no de la libertad ha sido planteado por la razón en el orden teórico y, por tanto, habrá de ser resuelto al menos en primera instancia exclusivamente dentro de él (A476-484/B504-512). La solución kantiana consistirá en afirmar que en las Antinomias dinámicas, en general, y en la Tercera Antinomia, en particular, tanto las posturas de las tesis como de las antítesis pueden ser verdaderas si se admite que, a diferencia de lo que ocurría con los miembros de las series de las Antinomias matemáticas, en las dinámicas pueden darse miembros heterogéneos entre sí (A529-530/B557558, A535-537/B563-565). La necesaria distinción que ha de hacerse entre las cosas consideradas como fenómenos (Erscheinungen) y como cosas en sí (Dinge an sich) constituye el elemento clarificador que permite compatibilizar mutuamente la tesis y la antítesis de cada una de las Antinomias dinámicas.

Pero, pese al evidente avance logrado en lo referente al proceso de legitimación de la atribución de realidad a la idea de libertad, no debe olvidarse que lo más que se ha logrado hasta este preciso momento ha sido una defensa fundamentada de su posibilidad lógica y no una prueba de su realidad, ni tan siquiera de su posibilidad real, “(...) puesto que, partiendo de la experiencia, nunca podemos inferir algo que no deba ser pensado según leyes de la experiencia" (A558/B586). Ahora bien, este avance resulta particularmente notable en lo relativo a las relaciones mantenidas entre la causalidad natural y la libertad, pues deja ver a las claras que "(...) naturaleza y causalidad por libertad no son incompatibles" (A558/B586).

La distinción entre fenómenos y cosas en sí plantea el tipo o modo de relación que ha de darse entre ellos dentro del marco abierto por el método idealista transcendental de filosofar. Varios intérpretes kantianos, entre los que se encuentra Allison, sostienen la posibilidad de atender a dos modelos interpretativos de esta distinción: la "interpretación de los dos mundos" y la “interpretación de los dos aspectos” (1990, pp. 3-4; 1996, pp. 3-4).

En la "interpretación de los dos mundos", la distinción toma la forma de diferenciación de índole esencial entre las entidades pertenecientes al mundo sensible y las que forman parte del mundo inteligible. El problema con el que se encuentra esta interpretación radica en que, si se recurre al concepto de "afección" para tratar de dar cuenta de la relación entre estos mundos y si ella es concebida en términos de causalidad natural, no se entiende cómo puede ser que un modo de relación que resulta constitutivo precisamente del mundo fenoménico pueda dar cuenta de una relación que se establece entre dos mundos, que por hipótesis son radicalmente distintos, donde además 
ocurre que es el mundo nouménico el que ha de llevar la iniciativa en el proceso de relación mutua ${ }^{3}$.

La "interpretación de los dos aspectos" evita esta dificultad mediante el procedimiento de considerar que la distinción entre fenómeno y cosa en sí no se establece entre dos conjuntos de entidades heterogéneas entre sí, sino que se trata de una diferenciación entre dos modos de abordar un único conjunto de entidades. De acuerdo con esta interpretación, los fenómenos son cualesquiera entidades mundanas consideradas en relación con las condiciones que hacen posible el conocimiento teórico humano (espacio y tiempo, categorías), mientras que las cosas en sí son esas mismas entidades, pero tomadas con independencia de tales condiciones. Ahora bien, como consecuencia del positivismo imperante y de la vigencia de la ciencia positiva en nuestra cultura, podrá siempre objetársele a este modelo interpretativo qué es lo que legitima, si es que lo hay, que pueda hablarse con fundamento de otros modos de consideración de las entidades mundanas distintos al de su conocimiento (y podríamos añadir) orientado a su manipulación técnica, constituyendo la libertad uno de tales modos de consideración bajo sospecha en nuestro tiempo.

Tal como Dal Monte expone, el problema con el que la "interpretación de los dos aspectos" se enfrenta estriba en que, considerando la distinción entre fenómeno y cosa en sí como una diferenciación entre diferentes aspectos de unas mismas entidades, ciertamente se evita la contradicción de sostener que lo que en un mundo se origina de manera necesaria, en otro lo pueda hacer libremente. Pero el precio que con ello se paga es la incapacidad para dotar de realidad a las propiedades de cada uno de estos ámbitos, de manera que tanto la libertad transcendental como la causalidad natural dejarían de ser "reales", al menos en el sentido habitual que a este término se le asigna (Dal Monte, 2019, pp. 492-495).

La cuestión que entonces se plantea es la de cómo deben ser caracterizadas tanto la causalidad natural como la causalidad mediante libertad ("transcendental")

\footnotetext{
3 Rivera de Rosales plantea en el capítulo segundo de su libro El punto de partida de la metafísica transcendental: un estudio crítico de la obra kantiana, y en el artículo "Die vierfache Wurzel des Dings an sich", un modelo alternativo a este esquema interpretativo, relativo a la distinción entre la cosa en sí y el fenómeno en la filosofía kantiana. Conforme a él, el problema de la cosa en sí resulta cuando el tema de la afección es abordado siguiendo un planteamiento precrítico, siendo en realidad lo que allí quiere ser pensado que el sujeto transcendental no crea ex nihilo la realidad del mundo, sino que ésta le tiene que estar dada.
} 
para que, sin abandonar su carácter "real" (lo que en el caso de la libertad supone no suscribir un modelo compatibilista suyo), puedan mantener en términos de efectividad una autonomía propia sin entrar en conflicto mutuo.

\section{Compatibilidad de la causalidad natural con la libertad transcendental}

La solución kantiana a la Tercera Antinomia ha planteado como compatibles la causalidad por libertad con la causalidad natural, sin caer para ello en el concepto de libertad utilizado en el "compatibilismo". El procedimiento que se sigue ha consistido en remitir la realidad de la libertad a un nuevo ámbito, como es el de la cosa en sí, que resulta compatible con el del fenómeno, aunque manteniendo que en este último no es compatible la causalidad por libertad con la causalidad natural. Lo que habrá que tratar de encontrar será, entonces, una forma en que pueda ser interpretada la categoría de causalidad natural, de manera que la realidad de la misma no entre en colisión con la posibilidad de la libertad transcendental.

Para llevar a cabo esta tarea, resulta necesario comenzar exponiendo de qué maneras admiten ser interpretada en el marco de la filosofía teórica kantiana la categoría de causalidad natural y la Segunda Analogía de la experiencia. Tomando como referencia el análisis que Allison lleva a cabo en varios pasajes de sus obras (1992, pp. 335-336, 344, 351-352, 355, 360-361, 492; 1996, p. $80)$, consideramos que pueden proponerse dos interpretaciones acerca del alcance y sentido de esta categoría y de su correspondiente Principio. El fundamento de la distinción entre ambas se encontraría en qué se estima que constituye el objeto de la defensa kantiana de la causalidad natural, si se trata de llevar a cabo una salvaguarda suya frente a la interpretación que Hume hizo de ella en la Investigación sobre el entendimiento humano (2004, pp. 181-183), o si más bien lo que se pretende es protegerla ante el ataque al que fue sometida en el Tratado de la naturaleza humana (2005, p. 138). Conforme con la primera, el objeto de crítica lo constituiría la presunta falta de legitimidad del paso por el que el efecto resulta inferido a partir de la causa empíricamente constatada, pudiéndose decir que lo puesto en entredicho sería la anticipación del futuro que se lleva a cabo mediante la causalidad natural. Mientras que atendiendo a la segunda, lo sometido a crítica de manera preferente habría sido más bien la legitimidad misma del proceso por el que son buscadas las causas que dan lugar a los efectos empíricamente constatados, es decir, lo ahora cuestionado resultaría ser más bien el proceso mismo de remontarse hacia el pasado partiendo del presente, que la inferencia que del futuro se hace tomando como referencia el presente. 
La primera interpretación admite ser calificada como una "interpretación fuerte". De acuerdo con ella, la Segunda Analogía de la experiencia establecería de qué tipo son las relaciones que hay en la naturaleza entre estados de cosas. Tales relaciones serían necesariamente relaciones de causalidad natural; mecanicista, según la interpreta Kant. Esta interpretación es una interpretación "ontológica", pues dice cómo son las relaciones entre las cosas. Ella supone un modelo interpretativo de corte realista, de acuerdo con el cual, por una parte, hay una naturaleza constituida según unas leyes no dependientes en su fundamento de la capacidad cognoscitiva humana y, por otra, un sujeto cognoscente dotado de sus propias leyes cognoscitivas entre las que se encuentra la ley de causalidad, dándose la "feliz" circunstancia de que ambas legalidades coinciden. Lo que así vendría a hacer la Segunda Analogía de la experiencia de acuerdo con esta interpretación, sería corroborar que también desde el punto de vista de las relaciones entre estados de cosas la naturaleza es regular. En relación con la temática de la realidad de la libertad que nos está ocupando en este artículo, lo relevante se encontraría en que, suscribiendo esta interpretación, habría de derivarse la presencia de un determinismo causal estricto en la naturaleza, pues conforme a ella no quedaría abierto espacio ontológico alguno para que pudiese darse otro tipo de relación entre las cosas de la naturaleza que no fuese el de la causalidad natural.

La segunda interpretación admite ser categorizada como "débil". Conforme a ella, la Segunda Analogía de la experiencia no establecería cómo es la naturaleza, sino cuál es la regla que debe seguirse en su investigación. A diferencia de lo que suponía la anterior interpretación, en este caso no se trataría de una interpretación "ontológica", sino de una "metodológica". Lo que la categoría de causalidad natural y la Segunda Analogía de la experiencia permitirían hacer, es convertir la sucesión subjetiva de representaciones en el psiquismo en una objetiva mediante el establecimiento de relaciones de causalidad natural entre estados de cosas. Enfocando la problemática desde una interpretación global de la filosofía teórica kantiana, lo que la Segunda Analogía haría es posibilitar uno de los modos (los otros son el resto de Principios) por medio de los cuales se irá "construyendo" la regularidad de la naturaleza (es decir, la experiencia), no procediendo a realizar, como se hacía en la anterior interpretación, una afirmación acerca de cómo sea la naturaleza (regular) con independencia de nuestras condiciones epistémicas (Duque, 1989, pp. 295-296). 
En apoyo a la interpretación débil de la Segunda Analogía de la experiencia, frente a su interpretación fuerte, pueden aportarse varios pasajes de la $\mathrm{KrV}$.

En primer lugar, en la Deducción transcendental de las categorías, Kant intenta justificar el carácter necesario que ellas poseen en su aplicación a la experiencia. La forma en que lleva a cabo tal intento consta de un doble movimiento, pues por una parte trata de mostrar que la multiplicidad sensible es unificada por medio de las categorías y, por otra parte, que sólo la aplicación de las mismas es capaz de dar lugar a la experiencia. La consecuencia de todo ello consiste en la constatación de que es nuestro propio entendimiento el que introduce la unidad necesaria en la multiplicidad sensible, por lo que Kant concluye que "(...) somos, pues, nosotros mismos los que introducimos el orden y regularidad de los fenómenos que llamamos naturaleza (Natur)" (KrV, A125-126), quedando como tarea pendiente para este sujeto que investiga la naturaleza, buscar la ley natural concreta según la cual la naturaleza se especifica en cada caso (A126-128). Él llevará a cabo esta misión bajo el supuesto realista de que la naturaleza actúa causalmente, pero si hubiese emprendido una investigación transcendental de tal supuesto, habría llegado a la conclusión de carácter idealista de que su origen no es otro que su propia actividad cognoscente.

En segundo lugar, en la Disciplina de la razón pura, Kant explicita el carácter que posee el principio de causalidad natural, enfatizando que él es un "principio" (Grundsatz) que hace posible la "experiencia" (Erfahrung) y no un "teorema" (Lehrsatz) acerca de esa misma "experiencia" (A736737/B764-765). No se trata, por tanto, de que tengamos un conocimiento " $a$ priori" de la naturaleza, sino de que por medio de la reflexión transcendental llegamos "a priori" al conocimiento de que en la construcción de la naturaleza (experiencia) procedemos precisamente haciendo uso de este principio, siendo este conocimiento el que luego aplicamos en la búsqueda de las leyes causales concretas, capaces de "deletrear" los sucesos naturales conjuntamente con el principio heurístico o regulativo de la finalidad en la naturaleza, analizado de manera temática por Kant en la $K U$.

En tercer lugar, la formulación kantiana correspondiente a la categoría modal de necesidad (Nothwendigkeit) y a su correspondiente Principio (Tercer Postulado del pensar empírico (A218/B266)) también habla a favor de esta interpretación. De acuerdo con ella, la necesidad no es algo que de suyo pertenezca como predicado a la existencia de las cosas, sino que ella es un modo de relación que se establece entre un evento (en este caso, una sucesión de estados) y el conocimiento de la naturaleza del que se dispone en ese 
momento. Por tanto, un evento se convierte en necesario cuando su "darse", que implica una observación o la conexión por medio de las leyes de la experiencia con una observación (categoría modal de realidad: Wirklichkeit), es puesto como deducible a partir de la experiencia con la que ya se cuenta.

La interpretación débil de la categoría de causalidad natural invalida la posibilidad ontológica del determinismo, pero de ello no puede concluirse el apoyo a las tesis que defienden el indeterminismo en la naturaleza. Afirmar el indeterminismo en ella supondría sostener que en algo (en una sucesión de estados observados) la naturaleza se muestra como no regular. Pero esto también constituye un dogmatismo equivalente al dogmatismo determinista. Lo ajustado a la interpretación débil del principio de causalidad natural que aquí se ha sostenido, consiste en decir que hasta ese preciso momento, las leyes causales empíricas conocidas en su desarrollo y aplicación no logran dar cuenta del evento (sucesión de estados) que se está considerando, pero que se plantea como tarea buscar nuevas leyes causales empíricas y/o aplicar (desarrollar) las leyes causales empíricas existentes con objeto de dar cabida dentro de la experiencia a ese evento concreto hasta entonces inasimilable.

Un ejemplo de que así es en realidad como procede la ciencia. históricamente lo encontramos en lo que ocurrió a principios del siglo XX, donde diversos experimentos a nivel microscópico (radiación del cuerpo negro, etc.) y a nivel macroscópico (experimento de Michelson-Morley, etc.) no pudieron ser explicados según la Física y la Geometría clásica (Física newtoniana, leyes de Maxwell, suposición del éter, Geometría euclidiana, etc.), lo que dio lugar al planteamiento de nuevas hipótesis (Planck, Lorentz) de las que resultaron la Física cuántica, la Teoría de la Relatividad (Especial, General) y las Geometrías no euclidianas. Ante la incapacidad explicativa de las antiguas leyes causales empíricas los científicos no pasaron a decir que la naturaleza no era regular, es decir, que no se encontraba sujeta al principio de causalidad, sino que siguieron enunciado teorías para tratar de dar cuenta de esa "aparente" carencia de regularidad según patrones de regularidad.

En esta misma línea pueden encuadrarse también los intentos llevados a cabo por Bohm más recientemente, tratando de suprimir el indeterminismo en la Física cuántica mediante la elaboración de una "teoría de variables ocultas" (Jammer, 1974, p. 255). Pese a que el Teorema de Bell suponga una seria objeción a la realizabilidad de este proyecto (p. 306), la limitación del alcance de este teorema, constatada por algunos científicos, (p. 312) y la defensa por parte de otros de la posibilidad de construir modelos matemáticos de algunos sistemas físicos que permiten implementar una teoría de variables ocultas 
de ellos (pp. 318-319), ponen de manifiesto que al menos buena parte de la comunidad científica elabora sus teorías guiada por la intención de que éstas han de ser deterministas, y que bajo esta intención subyace la común consideración de que la naturaleza de alguna manera permite ser analizada bajo algún patrón de índole determinista.

Estimamos por tanto que la interpretación débil de la Segunda Analogía de la experiencia aquí presentada, por ser más coherente que la fuerte con el sentido general que se ha presumido para el idealismo transcendental kantiano, así como por contar con suficientes apoyos en la obra filosófica kantiana, y encontrarse en consonancia con los desarrollos científicos modernos, constituye la interpretación preferible a la hora de proceder a caracterizar el sentido y el alcance que pueda concederse a la causalidad natural dentro del marco filosófico abierto por el modo transcendental de reflexión kantiano.

El principio de causalidad no debe, por tanto, ser visto como un "conocimiento a priori", sino como un "a priori" del conocimiento, es decir, como una regla que permite afirmar que se dispone de un conocimiento (Erkenntnis) y no simplemente de una opinión (Meinung) o una creencia (Glaube). De aquí que el falibilismo de Popper sólo tenga aplicación respecto de las leyes causales específicas (que pueden ser verdaderas o falsas) y no en relación con el principio de causalidad mismo, que siempre es verdadero, pues es constitutivo de la experiencia. Es más, aunque en un momento determinado todas las leyes causales específicas hubiesen sido refutadas, el principio de causalidad no perdería su validez, pues es un principio que indica el modo como debe procederse en la investigación para alcanzar un conocimiento objetivo y no un enunciado acerca de la manera de ser de un supuesto mundo externo a (independiente de) nuestras capacidades (facultades) cognoscitivas. Pero no por ello puede pasarse al extremo contrario, sosteniendo que la validez del principio de causalidad mismo no depende de que se constaten o no regularidades en la naturaleza, pues si nunca se hubiese constatado regularidad alguna en ella no nos encontraríamos en posesión de tal principio, lo cual significa en términos transcendentales que a su "idealidad transcendental" (transzendentale Idealität) le ha de corresponder también una "realidad empírica" (empirische Realität), consistente en su constatable eficacia a la hora de alcanzar conocimientos referidos a la naturaleza ${ }^{4}$.

\footnotetext{
4 Véase al respecto el capítulo primero de El punto de partida de la metafísica transcendental: un estudio crítico de la obra kantiana.
} 


\section{Compatibilidad de la libertad transcendental con la causalidad natural}

A lo largo del presente artículo estamos tratando de encontrar una formulación del concepto de causalidad natural como de la idea de libertad que, en la línea de la solución aportada por Kant al conflicto antinómico entre la causalidad natural y la libertad transcendental, los haga mutuamente compatibles. Para ello, no podemos dejar de atender a los resultados que la investigación científica nos proporciona y a lo que la lógica de la investigación científica nos revela acerca del modo como los científicos proceden en su consideración de la realidad. Hasta ahora hemos visto que era posible llevar a cabo una interpretación de la categoría de causalidad natural en términos de principio regulativo que "esquivase" las pretensiones omnicomprensivas, que para la causalidad natural se derivarían en el caso de que se suscribiese el determinismo científico como carácter definitorio acerca de la realidad, lo que permitiría abrir un espacio "ontológico" para la afirmación de la posibilidad de la libertad. En este apartado invertiremos el sentido en el que consideraremos la relación de compatibilidad que se busca establecer entre la causalidad natural y la libertad, mostrando que es posible defender una idea de la libertad que no invalide el carácter real que se atribuye a la causalidad natural en el encadenamiento de los sucesos del mundo. Para ello, mostraremos que el concepto "libertarianista" de libertad no puede ser suscrito, pues incluso los resultados aparentemente "anómalos" respecto de la imagen tradicional del mundo ofrecidos por la física cuántica, admiten ser interpretados en un sentido que no elimine la validez de la categoría de causalidad natural cuando pretenda darse cuenta de lo que acontece en el mundo natural.

El problema, y al mismo tiempo el reto que la Física cuántica plantea a la concepción determinista del mundo, asentada en el carácter veritativo y real del principio de causalidad natural elaborada a partir de la Física clásica es doble. Por un lado, sostiene que se da un entrelazamiento entre algunas magnitudes según las Relaciones de Incertidumbre de Heisenberg (momento y posición, por un lado, energía y tiempo, por otro), haciendo que el producto de los errores que se cometen al proceder a su medición simultánea, en ninguna situación pueda ser menor que un determinado valor constante (proporcional a la constante de Planck), por lo que nunca podrá llegarse a conocer completamente cuál es el estado de un sistema cualquiera. Por otro lado, las leyes que rigen las sucesiones de estados no establecen de manera única, tal y como hacían las leyes de la ciencia clásica, el estado que 
seguirá a un estado observado de un sistema, sino que se limitan a asignar las probabilidades correspondientes a cada uno de los posibles estados futuros. Estas dos características inducen a muchos a afirmar que la Física cuántica evidencia que la naturaleza es de suyo indeterminista.

La tentación en la que puede caerse desde una postura condicionada por la inclinación en favor de la afirmación de la realidad de la libertad, consiste en decir que efectivamente la Física cuántica refleja el carácter indeterminista de la naturaleza y que, si el determinismo es equivalente a la negación de la libertad en la naturaleza, el demostrado indeterminismo constituye una prueba irrefutable de que ya desde el punto de vista de la naturaleza (fenómenos) la libertad es real.

Un primer cargo de carácter general que puede objetarse a esta forma de proceder, es el de que la libertad toma su apoyo en la consideración de que no podemos decir qué estado seguirá a un estado dado de un sistema, pues en tal caso esto consistiría más bien en estar sometido al azar. Pero estar sometido al azar no es ser libre, sino que supone encontrarse absolutamente subyugado, pues entonces nada del futuro quedaría en nuestras manos.

Puesto que la moderna Física cuántica parece poner en duda la legitimidad de la aplicación del principio de causalidad natural, deberán ser sometidas a examen las formulaciones fuerte y débil anteriormente referidas de tal principio, con objeto de ver si alguna de ellas puede todavía seguir siendo coherente con el nuevo modo de considerar la naturaleza, introducido por los resultados de las investigaciones cuánticas.

La interpretación fuerte de la Segunda Analogía de la experiencia establece que causas similares producen efectos similares. Pero la Física cuántica no establece de manera única cuál será el estado que seguirá a un estado inicial dado, sino que se limita a indicar las probabilidades que corresponden a los estados futuros posibles. No establece entonces una única cadena de estados sucesivos que necesariamente tenga que darse, sino varias cadenas posibles, asociando a cada una de ellas una probabilidad. De esta manera, si la regularidad de la naturaleza se interpreta como un hecho, la Física cuántica constituiría un contraejemplo a este hecho de la regularidad de la naturaleza. Por ello, atendiendo a la interpretación fuerte del principio de causalidad natural, esta nueva modalidad de investigación de la naturaleza supondría la negación de la posibilidad de hacer ciencia, pues el requisito para poder hacerla conforme a esta interpretación es que la naturaleza efectivamente sea 
regular, situación que parecen contradecir algunos de los resultados a los que ha llegado la investigación cuántica.

Pasemos ahora a analizar lo relativo a la interpretación débil, según la cual todo efecto tiene alguna causa. En esta interpretación, la causalidad natural no procedería con carácter predictivo, sino meramente explicativo de lo que ha ocurrido. Esto significa que, al menos en su intención primigenia, la causalidad natural iría hacia atrás buscando la causa natural que ha hecho posible un evento o estado del sistema en cuestión, y no se ocuparía de buscar las consecuencias que de él se derivarían. Este requerimiento explicativo se satisface indicando que ese evento es uno de los posibles eventos que podrían darse a continuación de un determinado evento previo (encontrado por medio de la investigación científica) que se considera su causa, y que a la sucesión del uno al otro le correspondía una determinada probabilidad. La Física cuántica satisfaría de esta manera los requerimientos de la interpretación débil, pues explica el porqué de los eventos. Se trataría de una teoría causalista, ya que pone en relación eventos bajo la consideración de que unos son causas de otros, pero no determinista, debido a que únicamente alcanza a decir cuáles son los estados futuros y posibles del sistema, y a asignar a cada uno de ellos una probabilidad, sin atreverse a indicar con absoluta certeza cuál será el estado del sistema que sucederá al actual.

La confrontación de la interpretación fuerte de la Segunda Analogía de la experiencia con los resultados que ofrece la Física cuántica introduce un indeterminismo en el paradigma científico, que es consecuencia de la ignorancia en la que deja la constatación de que la naturaleza no es estricta y absolutamente regular. Conforme a ello, la explicación por libertad de la sucesión de estados de un sistema se encontraría al mismo nivel valorativo que cualquier otro tipo de explicación que pudiese aportarse, luego tampoco habría motivo racional para decantarse por ella frente a cualquier otra. Sin embargo, si se confronta la interpretación débil de la Segunda Analogía de la experiencia con los resultados que la Física cuántica presente, se llega a la conclusión de que la naturaleza es regular (explicación desde el presente hacia atrás), pero no es determinista (explicación desde el presente hacia adelante), pues no dice que una cadena de estados sucesivos sea la que deba darse necesariamente, sino que se limita a asignar probabilidades a las diferentes secuencias posibles de estados, dejando por ello espacio "ontológico" para la realización de la libertad al posibilitar que, en los términos en los que Moya se refiere (2017, p. 33), pueda darse por parte del sujeto un "control plural" sobre el curso de la acción. 
La interpretación aquí defendida de la causalidad natural hace más factible el modelo de naturaleza que Popper atribuye a la interpretación de Copenhague de la Mecánica cuántica, y que él mismo defiende ("modelo propensitivo") (1985, p. 29). Esto es así porque, según entiende Popper la interpretación de Copenhague de la Mecánica cuántica, la naturaleza sería de suyo determinista para los seguidores de esta interpretación, ocurriendo que ese determinismo quedaría "distorsionado" por efecto de las necesarias medidas que deben hacerse en los sistemas, las cuales, por otra parte, resultan imprescindibles para conocer el estado en que ellos se encuentran cuando se tiene la intención de hacer predicciones sobre su evolución, pudiéndose sostener por tanto que en tales situaciones el determinismo de la naturaleza quedaría "oculto" a los ojos de sus investigadores. Suscribiendo este modelo de naturaleza, que niega que en ella se dé un indeterminismo de carácter esencial a ella misma, la tarea de los científicos sería entonces la de tratar de reconducir esta aparente irregularidad de la naturaleza a patrones de regularidad. Al respecto, debe tenerse en cuenta que esta regularidad ya empieza a introducirse en la naturaleza desde el momento en que se asignan probabilidades a los distintos cursos de evolución que pueda tener un sistema. Esto es precisamente lo que ocurre cuando el módulo de la función de ondas resultante de resolver la ecuación de Schrödinger, en el caso de su aplicación en situaciones físicas concretas, se interpreta como distribución de probabilidades de los valores que puede tomar la variable física considerada en cada una de esas situaciones. Pero es que, además, cuando se pasa de la predicción de lo que podría ocurrir a la explicación de lo que efectivamente ha ocurrido, el carácter indeterminado de la evolución de los sistemas desaparece, pues ya podrá haber sido encontrada cuál ha sido la causa concreta que ha hecho que se haya producido previamente ese tránsito, y no otro, de lo posible a lo real. En la terminología cuántica, esta peculiaridad recibe la denominación de "reducción del paquete de ondas", consistiendo en la proyección de la función de onda asociada al sistema sobre uno de sus autovectores (el correspondiente a la magnitud que se está considerando), lo cual a su vez da lugar a la "indeterminación" de su magnitud complementaria, debido a que ésta no queda proyectada sobre ninguno de sus autovectores, motivo por el cual no puede tomar ningún valor concreto (Penrose, 1991, pp. 315-317).

Moya presenta un instructivo ejemplo en el que, sin tener que recurrir a los complejos resultados experimentales de la Física cuántica, queda puesto de manifiesto cómo la introducción del concepto de probabilidad en la explicación de los fenómenos naturales no elimina el concepto de causalidad 
en el proceso de justificar su surgimiento. De acuerdo con esta intención, propone pensar el caso del lanzamiento de una piedra contra un vidrio. Como consecuencia del impacto puede ocurrir que el vidrio se rompa o que no lo haga (tal vez debido a la existencia de fenómenos cuánticos), pudiéndose asignar una probabilidad a cada uno de estos dos sucesos. Si el vidrio se rompe, queda claro entonces que ha sido el impacto de la piedra contra el mismo el causante de dicha rotura. De todo ello concluye que "(...) aunque había alguna probabilidad de que el vidrio no se rompiera, la rotura no fue, sin embargo, un fenómeno azaroso, un mero producto del azar, sino que tuvo una causa" (Moya, 2017, p. 144).

El ámbito del fenómeno queda constituido en la filosofía transcendental kantiana como el lugar de manifestación de las cosas en tanto que son cognoscibles teóricamente, y precisamente por serlo así. En este ámbito no es posible ningún otro tipo de causalidad que no sea la causalidad natural, pues considerar otro tipo de causalidad no sometida a las leyes naturales supondría la imposibilidad del conocimiento teórico objetivo, lo que estaría en contradicción lógica con la definición del ámbito del fenómeno como ámbito del conocimiento teórico objetivo (criterio de demarcación). Algunos de los encadenamientos de fenómenos, sucesos, que se producen en este ámbito son susceptibles de ser explicados recurriendo al ámbito de la cosa en sí (realidad en sí) por medio de la causalidad por libertad. Pero si, como se hace, la distinción entre fenómeno y cosa en sí no se interpreta como una distinción entre entidades distintas, sino como diferentes modos de considerar unas mismas entidades, es preciso indagar de qué modo particular, si es que lo hubiera, podría darse en el ámbito fenoménico un encadenamiento entre fenómenos cuando en él interviniese la causalidad por libertad. Una manera de llevar a cabo esta indagación puede ser distinguiendo -conforme Allison lo hace a partir de la indicación kantiana de que "(...) aunque el suceso sigue a estas causas naturales, no se sigue de ellas" (KrV, A450/B478)entre fenómenos que siguen (follow, folgen) a sus condiciones naturales antecedentes y aquéllos otros que surgen (arise, erfolgen) a partir de ellas (Allison, 1990, p. 26). Así, en el caso de los sucesos debidos a la causalidad por libertad, si se los considera desde el lado del fenómeno vendrían a continuación de sus fenómenos antecedentes, pero no se originarían a partir de ellos (cuya consideración es el objeto de estudio de la investigación científica), sino teniendo su origen en una causalidad por libertad, que a diferencia de las anteriores no podrá ser abordada desde el punto de vista científico. De esta manera, en el caso de los sucesos debidos a la causalidad 
por libertad, no sólo no quedaría rota con ella la legalidad de la naturaleza, sino que en ella misma sería posible constatar los efectos de esa causalidad por libertad, por lo que:

(...) no causa ningún perjuicio al entendimiento el admitir, aunque sólo fuera ficticiamente, que alguna de las causas naturales posea una facultad que sea sólo inteligible, en el sentido de que su determinación a la acción nunca se base en condiciones empíricas, sino en meros fundamentos del entendimiento, pero de suerte que la acción de esa causa en la esfera del fenómeno sea conforme a todas las leyes de la causalidad empírica (allen Gesetzen der empirischen Kausalität gemäß sei) (KrV, A545/B573).

Este modelo explicativo aquí defendido acerca del modo como se manifiesta en el mundo la causalidad por libertad, no ha de suponer en ningún caso la defensa de que la libertad tenga una intervención en el mundo que "rompa", ni tan siquiera que suspenda, la vigencia de las leyes naturales, como resultaría de aceptar un concepto "libertarianista" de la libertad transcendental. Este modelo simplemente pretende resaltar que, haciendo uso de los términos que Moya emplea (2017, p. 33), resulta preciso complementar el espacio que la resolución de la Tercera Antinomia de la $\mathrm{KrV}$ ha dejado abierto, permitiendo ejercer un "control de origen o fuente" sobre la acción, con la posibilidad de que también se dé un cierto margen (holgura) en la naturaleza, que admita llevar a efecto un "control plural" sobre ella.

Si se admite que la relación entre realismo transcendental e idealismo transcendental, en lo que respecta a la cuestión del conocimiento, es una disyunción exclusiva, la solución dada a la Primera y a la Segunda Antinomia por medio de la distinción entre fenómeno y cosa en sí constituye una prueba indirecta de la verdad del idealismo transcendental en cuanto teoría cognoscitiva, lo que contribuye a aumentar la verosimilitud del idealismo transcendental considerado en su globalidad; en el caso que nos concierne respecto de la afirmación de la realidad objetiva de la libertad. La solución presentada para la Tercera Antinomia no supone una prueba de la verdad del idealismo transcendental en su globalidad, sino que sólo contribuye a aumentar su verosimilitud, pues únicamente logra establecer la posibilidad de la libertad como realidad en el ámbito de la cosa en sí y no alcanza nunca a poder afirmar su realidad objetiva, ya que en esta Antinomia se habla exclusivamente del ámbito de la cosa o realidad en sí como una perspectiva o punto de vista (ein Standpunkt), que es posible tomar (posibilidad) sin que se pueda llegar en ningún caso a la demostración teórica del mismo (realidad). Pero podría ocurrir que si en algún otro orden distinto del teórico 
se encontrase una prueba válida de la "realidad" de la libertad, la afirmación de esa libertad como propiedad del ámbito de la cosa en sí (realidad en sí) sería una prueba indirecta de la verdad del idealismo transcendental en su globalidad. Esta verdad consistiría, básicamente, en la atribución de realidad al ámbito de la cosa en sí, y su caracterización como ámbito autónomo (leyes propias) respecto al del fenómeno. Esta tarea será llevada a cabo por Kant en la $K p V$, mediante la apelación a la existencia de un Faktum der Vernunft que configura a la moralidad como ratio cognoscendi de la libertad, es decir, de su realidad práctica, y a la libertad como ratio essendi de la moralidad.

\section{Conclusión}

La relación mutua de carácter conflictivo entre el concepto de causalidad natural y la idea de libertad, que constituye gran parte del pensamiento contemporáneo, ya fue abordada por Kant en la Tercera Antinomia de la $K r V$. La solución kantiana a esta contradicción, en la que la razón parece encontrarse sumida, consistió en la defensa de la posibilidad (lógica) de la libertad transcendental, concebida como la capacidad para dar lugar a una nueva serie de sucesos en el mundo, pero sin que ello hubiese de suponer la pérdida de vigencia de las leyes naturales, que se consideran como las únicas que pueden explicar el modo como se produce la sucesión temporal de estados del mundo. Kant da cuenta de la posibilidad de concurrencia de ambos modelos de causalidad en el mundo mediante su conocida distinción entre fenómenos y cosas en sí. Pero, con objeto de disipar la duda que podría plantearse en relación con el posible carácter ad hoc que esta distinción pudiese tener, y sin intención de entrar en el marco práctico de consideración de la posible realidad que pudiese asociarse a la idea de libertad, se ha pretendido en el presente artículo ofrecer una caracterización tanto de la causalidad natural como de la libertad, que posibilite su concurrencia y sea compatible con la imagen que del mundo natural ofrece la ciencia moderna.

La presunta incompatibilidad entre la categoría de causalidad y la libertad que resultaría de sostener posiciones de índole determinista, desaparece si se procede interpretando la causalidad natural como un principio de índole regulativa que ha de guiar el modo como la investigación natural debe ser llevada a efecto y no como un principio constitutivo de la naturaleza misma con independencia del modo como ella sea conocida. Esta interpretación del alcance de la categoría de causalidad natural no sólo se revela coherente con la imagen acerca de la naturaleza que proporciona la Física clásica, sino también al menos con gran parte de los resultados otorgados por la moderna Física cuántica. 
No resulta por ello necesario el recurso a una interpretación de carácter "libertarianista" acerca del modo como la libertad pueda hacerse realidad en el mundo natural, sino que es posible concebir que el "necesario" encadenamiento causal en éste puede presentar un margen que posibilite la acción en él de una causalidad de carácter libre, tal como Kant mismo pudo haber reconocido mediante la distinción entre estados que "surgen" a partir de sus estados anteriores y aquéllos otros que meramente les "siguen". Pero, pese a las posibilidades que esta hipótesis acerca del alcance y ámbito de validez del principio de causalidad natural abre en relación con la cuestión de la realidad de la libertad, las consideraciones que pueden hacerse en el ámbito especulativo de reflexión se revelan insuficientes, por lo que de cara a su dilucidación definitiva será preciso atender también a las de índole práctica que Kant realiza en obras como la $\mathrm{KpV}$.

\section{Referencias}

Allison, H. E. (1990). Kant's theory of freedom. Cambridge: Cambridge University Press.

Allison, H. E. (1992). El idealismo transcendental de Kant (D. Granja Castro, Trad.). Barcelona: Anthropos.

Allison, H. E. (1996a). Causality and causal law in Kant: A critique of Michael Friedman. Idealism and freedom. Essays on Kant's theoretical on practical philosophy (pp. 80-91). Cambridge: Cambridge University Press.

Allison, H. E. (1996b). Transcendental idealism: a retrospective. Idealism and freedom. Essays on Kant's theoretical and practical philosophy (pp. 3-26). Cambridge: Cambridge University Press.

Dal Monte, D. (2019). The epistemological interpretation of transcendental idealism and its unavoidable slide into compatibilism. Revista de Estudios Kantianos, 4 (2), pp. 476-507. https://doi.org/10.7203/ REK.4.2.13939

Duque, F. (1989). Causalidad y teleología en Kant. J. Muguerza y R. Rodríguez Aramayo (Eds.), Kant después de Kant. En el bicentenario de la Crítica de la razón práctica (pp. 285-307). Madrid: Tecnos. 
Hume, D. (2004). Investigación sobre el entendimiento humano (V. Sanfélix y C. Ors Marqués, Trads.). Madrid: Istmo.

Hume, D. (2005). Tratado de la naturaleza humana (F. Duque, Trad.). Madrid: Tecnos.

Jammer, M.(1974). The philosophy of quantum mechanics. The interpretations of quantum mechanics in historical perspective. New York: John Wiley \& Sons Inc.

Kant, I. (1989). Crítica de la razón pura (KrV) (P. Ribas, Trad.). Madrid: Editorial Alfaguara.

Kant, I. (2000). Kant im Kontext 2000. Teilausgabe I. Werke, Briefwechsel und Nachlaß auf CD-ROM (Citada como $A A$ ). Berlin: Karsten Worm InfoSoftWare.

Laplace, P. S. (1985). Ensayo filosófico sobre las probabilidades (P. Castrillo, Trad.). Madrid: Alianza.

Moya, C. (2017). El libre albedrío. Un estudio filosófico. Madrid: Cátedra.

Penrose, R. (1991). La nueva mente del emperador (J. J. García Sanz, Trad.). Barcelona: Grijalbo Mondadori.

Popper, K. (1985). Post Scriptum. Volumen III (M. Sansigre, Trad.). Madrid: Tecnos.

Rivera de Rosales, J. (2011). El punto de partida de la metafísica transcendental: un estudio crítico de la obra kantiana. Madrid: Xorki.

Rivera de Rosales, J. (2013). Die vierfache Wurzel des Dings an sich. S. Bacin; A. Ferrarin; C. La Rocca und M. Ruffing (Eds.), Kant und die Philosophie in weltbürgerlicher Absicht. Akten des XI. Internationalen Kant-Kongresses (pp. 743-753). Berlin: Walter de Gruyter.

Spinoza, B. (2007). Ética demostrada según el orden geométrico (V. Peña, Trad.). Madrid: Alianza. 